Analysis of Conductor Impedances Accounting for Skin Effect and Nonlinear Permeability

M. P. Perkins, M. M. Ong, C. G. Brown, R. D. Speer

July 22, 2011

18th IEEE International Pulsed Power Conference Chicago, IL, United States June 19, 2011 through June 23, 2011 
This document was prepared as an account of work sponsored by an agency of the United States government. Neither the United States government nor Lawrence Livermore National Security, LLC, nor any of their employees makes any warranty, expressed or implied, or assumes any legal liability or responsibility for the accuracy, completeness, or usefulness of any information, apparatus, product, or process disclosed, or represents that its use would not infringe privately owned rights. Reference herein to any specific commercial product, process, or service by trade name, trademark, manufacturer, or otherwise does not necessarily constitute or imply its endorsement, recommendation, or favoring by the United States government or Lawrence Livermore National Security, LLC. The views and opinions of authors expressed herein do not necessarily state or reflect those of the United States government or Lawrence Livermore National Security, LLC, and shall not be used for advertising or product endorsement purposes. 


\title{
Analysis of Conductor Impedances Accounting for Skin Effect and Nonlinear Permeability
}

\author{
M.P. Perkins, M.M. Ong, C.G. Brown Jr., R.D. Speer \\ Lawrence Livermore National Laboratory \\ 7000 East Avenue \\ Livermore, CA 94550 USA
}

\section{Abstract}

It is often necessary to protect sensitive electrical equipment from pulsed electric and magnetic fields. To accomplish this electromagnetic shielding structures similar to Faraday Cages are often implemented. If the equipment is inside a facility that has been reinforced with rebar, the rebar can be used as part of a lighting protection system. Unfortunately, such shields are not perfect and allow electromagnetic fields to be created inside due to discontinuities in the structure, penetrations, and finite conductivity of the shield. In order to perform an analysis of such a structure it is important to first determine the effect of the finite impedance of the conductors used in the shield. In this paper we will discuss the impedances of different cylindrical conductors in the time domain.

For a time varying pulse the currents created in the conductor will have different spectral components, which will affect the current density due to skin effects. Many construction materials use iron and different types of steels that have a nonlinear permeability. The nonlinear material can have an effect on the impedance of the conductor depending on the B-H curve. Although closed form solutions exist for the impedances of cylindrical conductors made of linear materials, computational techniques are needed for nonlinear materials. Simulations of such impedances are often technically challenging due to the need for a computational mesh to be able to resolve the skin depths for the different spectral components in the pulse. The results of such simulations in the time domain will be shown and used to determine the impedances of cylindrical conductors for lightning current pulses that have low frequency content.

\section{INTRODUCTION}

Lightning is composed of current pulses which can be appreciable on the millisecond time scale and derivatives on the microsecond time scale. For facilities made with good conductors that have little loss, the E-field in the facility will be mainly inductive and the B-field will be related to the current. Thus, for objects that behave as small monopole and loop type antennas [1], [2], the voltages at the terminals of the antennas will be related to the derivative of the current. Two current waveforms commonly used to approximate lightning, the Double Exponential and the Heidler, were used to represent an extreme (1\%) negative lightning strike that satisfies the parameters discussed in [3]. Their current waveforms, derivatives, and spectrums are shown in figure 1.
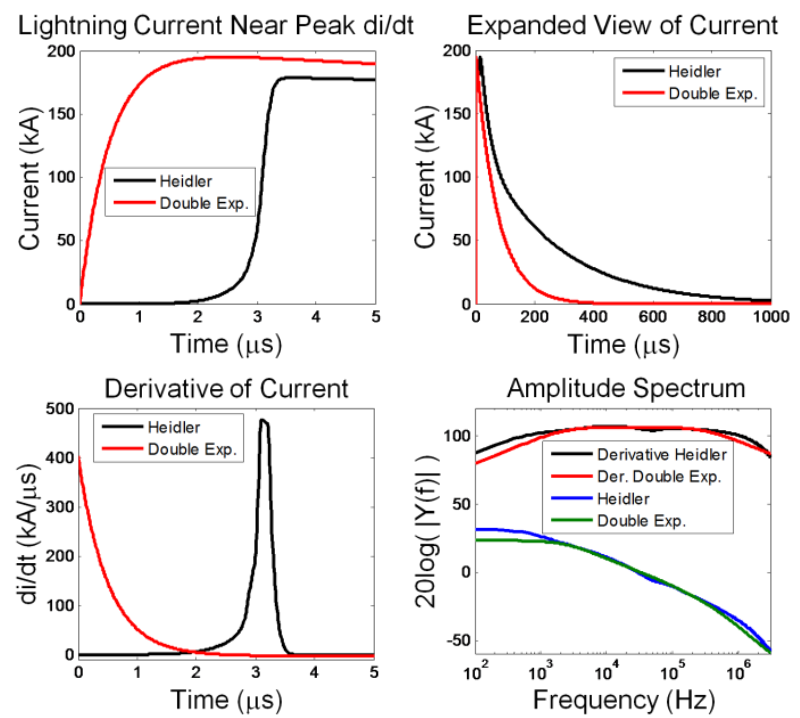

Figure 1. Zoomed in and expanded plot of lightning current as well as its derivative and amplitude spectrum.

This work performed under the auspices of the U.S. Department of Energy by Lawrence Livermore National Laboratory under Contract DE-AC52-07NA27344.

email: perkins22@llnl.gov 
Figure 2 illustrates the peak E-fields and B-fields developed at the center of a small facility that has a penetration which has been struck by lightning. The rebar of the facility, modeled as PEC for this example, creates a lightning protection system. For the two figures on the left, the penetration attaches to the right side of the facility and is not connected to the rebar where it enters the facility. For the two figures on the right, the penetration is attached to the rebar structure using a bond wire where it enters the facility on the left and also attaches to the right side of the facility. The fields in the structure were significantly reduced by adding the bond wire. This reduction in fields is beneficial in lowering the coupling to electrical equipment inside the facility that may behave as small monopole and loop type antennas.

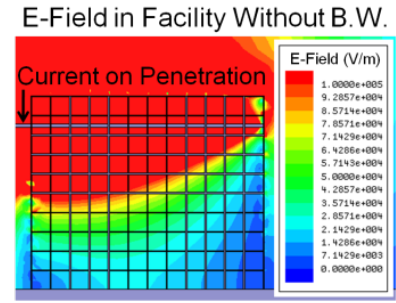

B-Field in Facility Without B.W.

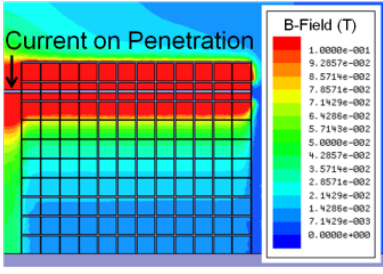

Figure 2. E-fields and B-fields developed in a small facility when a penetration is struck by lightning for examples without and with a bond wire.

In order to accurately determine the fields inside a facility that has been struck by lightning, the importance of the internal impedances of the conductors used in the lighting protection system must first be determined. Because rebar is commonly made of materials that have non-linear B-H curves, such as steel, computational techniques are needed in order to find the internal impedances. The problem is further complicated due to the diffusion of the E- and B-fields into the conductors.

\section{METHODOLOGY}

The voltage for a section of rebar or bond wire consists of time changing inductive and resistive components as shown in Eqs. (1) and (2).

$$
\begin{aligned}
& v(t)=d / d t\left[i(t) L_{\text {total }}(t)\right]+i(t) R(t) \\
& L_{\text {total }}(t)=L_{\text {internal }}(t)+L_{\text {external }}
\end{aligned}
$$

The total inductance for the section of conductor given by Eq. (2) consists of a constant external inductance as well as the time changing internal inductance. In order to determine the time changing resistance and internal inductance Maxwell's equations must be solved. From the spectrum for the lightning pulses shown in Fig. 1 we can see that lightning is a low frequency phenomena, thus low frequency approximations can be made. The low frequency form of Maxwell's equations to solve is given by Eqs. (3)-(6).

$$
\begin{aligned}
& \nabla \times E(x, t)=-\partial B(x, t) / \partial t \\
& \nabla \times H(x, t)=J(x, t) \\
& J(x, t)=\sigma E(x, t) \\
& B(x, t)=\mu_{o} \mu_{r}(H(x, t)) H(x, t)
\end{aligned}
$$

In Eq. (4) the displacement current has been neglected due to the low frequency approximation. We also see that the relationship between $\mathrm{B}$ and $\mathrm{H}$ is given using the nonlinear permeability. We will model the rebar as steel 1008 with a diameter of 0.75 ”. This is representative of rebar commonly used in many facilities. We will assume that the bond wire is made of copper with a diameter of 0.162". The finite element code Maxwell 3D was used to solve Eqs. (3)-(6) in the time domain [4], [5].

Using the results from Maxwell, the resistance can be computed from the relation between power dissipation in field form and lumped element form given by Eq. (7). Similarly, the internal inductance can be found from the relation of magnetic energy in field form and lumped element form as given by Eq. (8). In Eqs. (7) and (8) the volume integral is over the volume of the conductor and the surface integral is over the cross section of the conductor. The internal inductance was also calculated using the flux linkage as shown in Eq. (9). Good agreement was found between the results using Eqs. (8) and (9), with the percent difference reaching a maximum of $\sim 10 \%$ for the cases modeled, presumably due to the simplified integration scheme implemented for Eq. (9).

$$
\begin{aligned}
& R(t)=P_{d}(t) / i^{2}(t)= \\
& \iiint E(x, t) \cdot J(x, t) d V /\left(\iint J(x, t) \cdot d s\right)^{2} \\
& L_{\text {internal }}(t)=2 W_{m}(t) / i^{2}(t)= \\
& \iiint B(x, t) \cdot H(x, t) d V /\left(\iint J(x, t) \cdot d s\right)^{2} \\
& L_{\text {internal }}(t) i(t)=\Lambda(t)=\int_{0}^{a} B(r, t) i^{\prime}(r, t) / i_{\text {tot }}(t) d r= \\
& \int_{0}^{a} d r B(r, t) \int_{0}^{r} \rho J(\rho, t) d \rho / \int_{0}^{a} \rho J(\rho, t) d \rho
\end{aligned}
$$


Because of the nonlinear relationship between $\mathrm{B}$ and $\mathrm{H}$ Eqs. (7)-(9) are nonlinear. The nonlinear B-H curve for steel 1008 is shown in Fig. 3. The permeability is given by the slope of the B-H curve. For large $\mathrm{H}$-fields the value saturates to approximately that of free space, whereas for small $\mathrm{H}$-fields a large value of permeability results. We will refer to conductors that have the conductivity of steel 1008 with $\mu_{r}=1.03$ throughout the entire conductor as saturated and with $\mu_{r}=1206$ throughout the entire conductor as unsaturated. Also shown Fig. 3 are the skin depths, given by $\left(\pi f \sigma \mu_{o} \mu_{r}\right)^{-0.5}$, over the lightning frequencies. The conductivities of copper and steel 1008 are 5.8E7 S/m and 2E6 S/m. The skin depth for steel 1008 will be bounded by the saturated and unsaturated cases. One challenge to overcome in modeling the problem is to resolve the large variation in skin depth that occurs during lightning pulses.
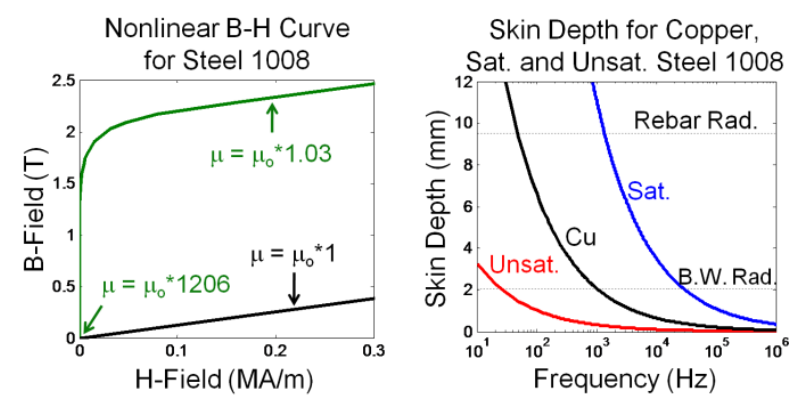

Figure 3. Nonlinear B-H curve for steel 1008 as well as skin depths for copper, saturated steel, and unsaturated steel over the frequencies of interest for lightning pulses.

The skin effect is illustrated in Fig. 4 in the frequency domain at $100 \mathrm{~Hz}$ and $1 \mathrm{MHz}$ for the saturated case. We can see that for $100 \mathrm{~Hz}$ the current density is uniform throughout the conductor but at $1 \mathrm{MHz}$ the current density has been moved to the outside of the conductor due to the skin effect. Because resistance is related to the amount of area the current density occupies, the low frequency case will have a lower resistance than the high frequency case. The H-field is related to the amount of current density it encloses. For the low frequency case the $\mathrm{H}$-field increases as $r$ up to the radius then decreases as $r^{-1}$. For the high frequency case the $\mathrm{H}$-field is pushed out of the conductor due to the skin effect. Because the internal inductance is related to the amount of magnetic energy inside the conductor, the low frequency case will have a higher inductance than the high frequency case. We will see in Sect. IV that it is convenient to use frequency domain methods for linear materials to determine the response of the conductor to lighting pulses.

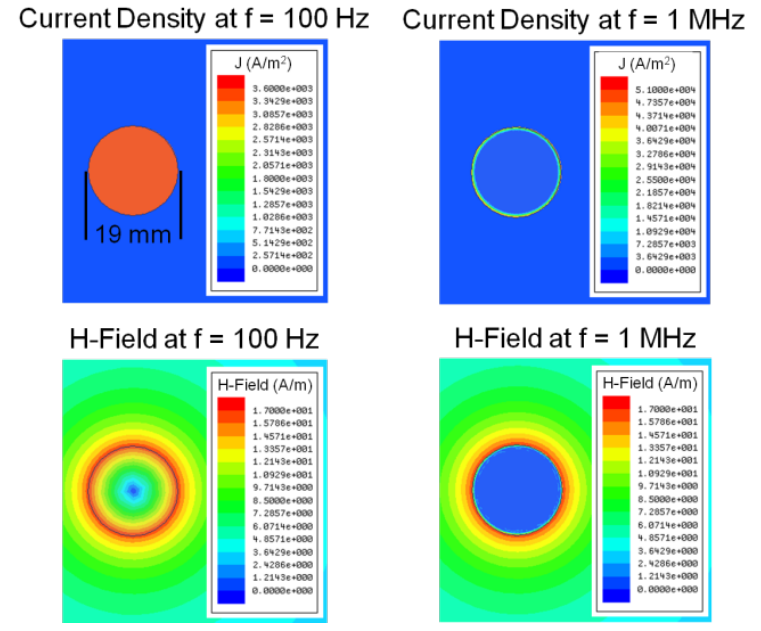

Figure 4. Current Density and H-field for 1 amp of current at $100 \mathrm{~Hz}$ and $1 \mathrm{MHz}$ for saturated steel.

In order to resolve the skin depths over the frequencies of interest eight nested cylinders were used. The finite element code Maxwell will ensure that each cylinder is meshed. This gives us more control over the initial mesh. The difference between the outer two cylinders was 40 $\mu \mathrm{m}$. The large scale difference between the $40 \mu \mathrm{m}$ spacing and the $19 \mathrm{~mm}$ diameter leads to a computationally intensive problem.

The computational mesh was created in the frequency domain by first solving at one frequency. The frequency was then changed and the mesh that was created for the initial frequency was used as the input mesh for the next frequency. This mesh was refined $30 \%$ and used to solve the problem. Then a different frequency was simulated using the previous mesh as the input mesh, which was once gain refined $30 \%$. This process was then repeated over the frequencies of interest for lightning pulses. This mesh that was created in the frequency domain was then used for time domain simulations. The algorithm discussed above for creating the mesh was critical in order to achieve accurate results. Without such a procedure, the mesh would not be resolved fine enough to be able to resolve the skin depths in the time domain and large errors could result due to the large range of skin depths.

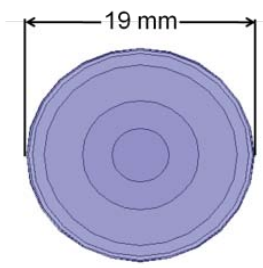

Figure 5. Eight nested cylinders used to model rebar sized cylindrical conductors. 


\section{FINITE ELEMENT RESULTS}

The procedure discussed above for creating the finite element mesh can be tested by comparing conductor impedances for frequency and time domain simulations. For the frequency domain simulations the nested cylinders were not used and the mesh was only refined using the one frequency the problem was solved at. This leads to an independent mesh than that used for the time domain simulations. In the time domain a current waveform of $\sin (2 \pi f t)$ was used as the input. The internal inductance and resistance was calculated for each case. The results are shown in Fig. 6. In Fig. 6, rebar sized cylinders were used and simulated for copper, the saturated and unsaturated steel cases, as well as the copper bond wire. The maximum percent difference over the entire band was less than $8 \%$, giving us confidence in the mesh created in the time domain.
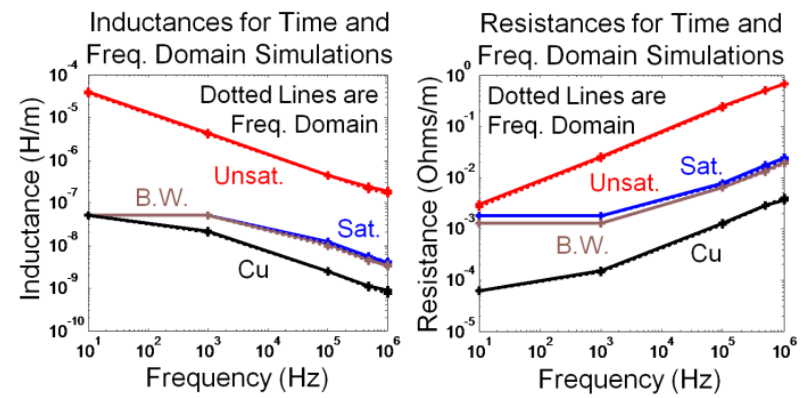

Figure 6. Comparison of internal inductances and resistances for a cylindrical conductor. The dotted lines (on top of solid lines) are frequency domain results and the solid lines are time domain results.

Because of the good agreement in impedances over the frequencies of interest, we have confidence to perform time domain simulations. Using the mesh created for the time domain, the Double Exponential and Heidler lighting current waveforms shown in Fig. 1 were applied as the current on a rebar sized cylinder. The resistances and inductances were computed using Eqs. (7)-(9). The results are shown in Fig. 7. The cases marked steel use the nonlinear B-H curve for steel 1008 shown in Fig. 3. For these simulations it was important that the lighting current had zero current at time zero. Some waveforms used to represent lightning have a small nonzero current at time zero. This introduces an initial condition of the conductor having stored energy, which can have an effect on the results. The nonphysical derivative for the Double Exponential (not zero at time zero) appears to have had a negligible effect on the results.
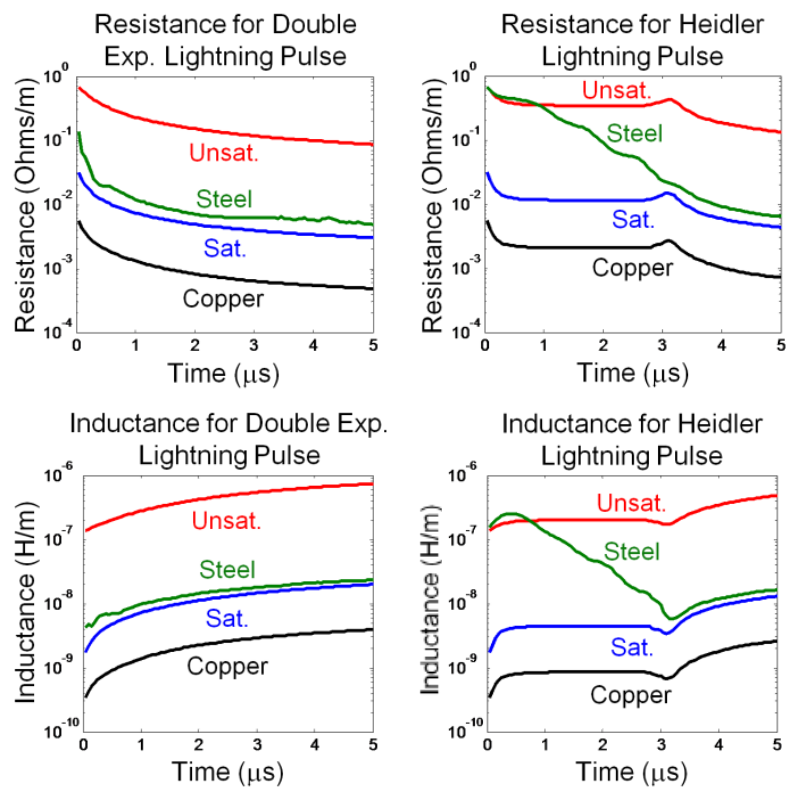

Figure 7. Time changing resistances and inductances calculated for a rebar sized conductor excited by Double Exponential and Heidler lighting current waveforms.

In Fig. 7 we can see that for the Double Exponential excitation the resistance decreases as time increases. This is because the Double Exponential has a large amount of current earlier in the pulse and fields diffuse into the conductor. The diffusion causes more of the cross sectional area of the conductor to be occupied by the current density, hence decreasing the resistance. The diffusion of fields into the conductor results in more internal magnetic energy, which is why the inductance increases. We can also see that the nonlinear case is close to the saturated case. Inside the conductor of steel the nonlinear permeability will change with radius depending on how far the fields have diffused into the conductor. We can see that the behavior of the impedances for the Heidler excitation is similar to that of the Double Exponential after there is appreciable current. However, earlier in time the Heidler has much lower current and a lower derivative. This is why the steel saturates slower for the cases with the Heidler excitation.

The impedances are used to calculate the resistive voltages $((i(t) R(t))$ and internal inductive voltages $\left(d / d t\left[i(t) L_{\text {internal }}(t)\right]\right)$. The results are shown in Fig. 8. We see from Fig. 8 that near the peak voltages the nonlinear steel cases are approximately the same as the saturated cases. The results for the nonlinear steel case can be made "smoother" by adjusting the mesh, time step, and nonlinear residual of the solver. Unfortunately this will result in longer simulations that require more memory. 


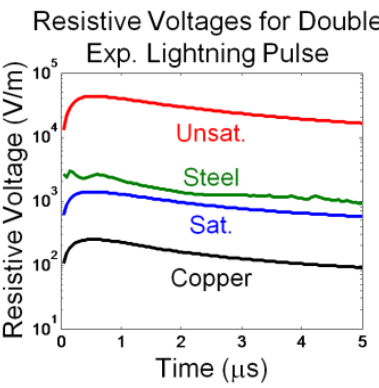

Inductive Voltages for Double
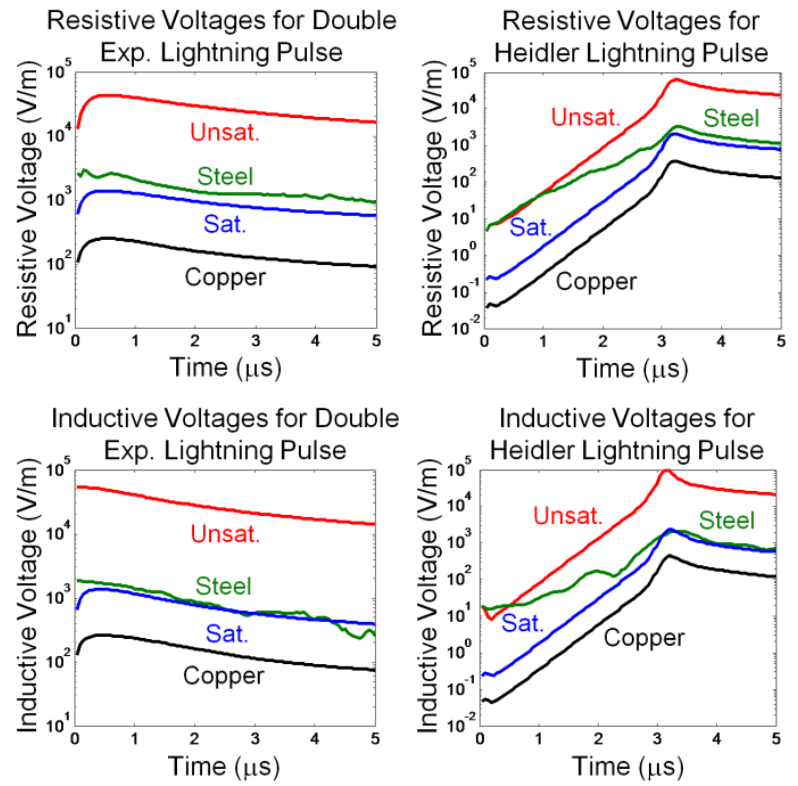

Figure 8. Resistive and internal inductive voltages calculated for a rebar sized conductor excited by lighting.

The total internal voltages for the unsaturated and nonlinear steel cases as well as the external inductive voltages are shown in Fig. 9. In the figure the solid lines are for the Heidler pulse and the dashed for the Double Exponential. A representative external inductance of $1 \mathrm{E}-6 \mu \mathrm{H} / \mathrm{m}$ was chosen. We can see that if the rebar does not saturate the internal voltages is on the same order as the external voltage. However, because of the nonlinearity in steel the internal voltage is much less than the external voltage. It is also interesting to note that although the derivative of the current goes to zero, the inductive contribution cannot be neglected because of $i(t) d L_{\text {internal }}(t) / d t$. This can be seen by comparing the external voltages in Fig. 9 and the inductive voltages in Fig. 8, which are appreciable even for small $d i(t) / d t$. Accounting for current division in the facility analysis and the effect of concrete is actively under investigation.

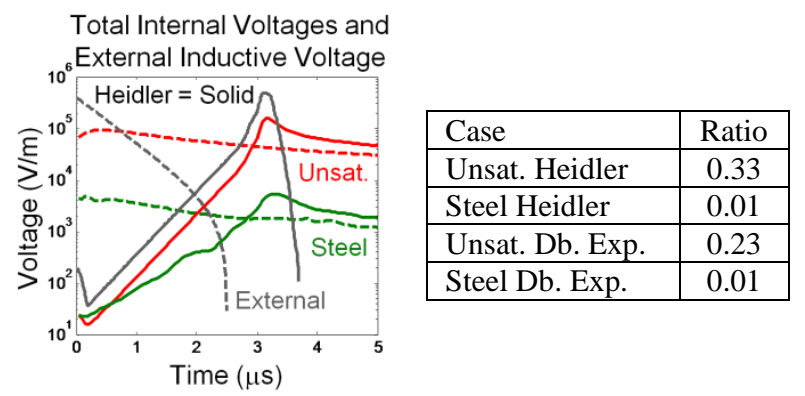

Figure 9. Total internal voltages and external inductive voltages, as well as the ratio between the peak total internal voltage and peak external voltage.

\section{TEMPERATURE ESTIMATIONS}

The internal impedance of conductors in the time domain due to pulse excitations has been investigated by other researchers for linear materials [6]-[10]. In [7]-[10] solutions specifically for cylindrical conductors were found. For cylindrical conductors made with linear materials Eqs. (3)-(6) can be reduced to yield Eq. (9) for the current density. By taking the Laplace transform of Eq. (9) the solution in frequency space can be found and is given by Eq. (10) [7]-[10].

$$
\begin{aligned}
& \partial^{2} J(r, t) / \partial r^{2}+\partial J(r, t) / r \partial r=\sigma \mu_{o} \mu_{r} \partial J(r, t) / \partial t \\
& J(r, s)=I(s) j k J_{o}(j k r) / 2 \pi a s J_{1}(j k a)
\end{aligned}
$$

In Eq. (10), $s$ is the Laplace variable, $I(s)$, is the Laplace transform of the current pulse, $J_{o}$ and $J_{1}$ are Bessel functions of the first kind of orders zero and one, $a$ is the radius of the conductor, and $k=\left(s \sigma \mu_{o} \mu_{r}\right)^{0.5}$. It is interesting to note that $k$ in Eq. (10) is related to the inverse of the skin depth.

A program was written to compute Eq. (10) then inverse transform back into the time domain. After solving for $J(r, t), H(r, t)$ was found. The resistances and inductances were then found for rebar sized cylinders made of copper, and the unsaturated and saturated steel cases using Eqs. (7) and (8). The radial spacing used in the program was nonlinear so that the skin depth could be resolved. Excellent agreement was found with the finite element results for copper and saturated steel. There were some slight oscillations for the unsaturated steel case due to the need to evaluate large argument Bessel functions, however good overall agreement was still achieved.

Using the computed current density found from the transform method discussed above, one can find the energy dissipated in the conductor by integrating the power dissipation over the time of the pulse. One can use this to obtain a rough estimate of the temperature rise at the surface of the conductor using Eq. (11) [8]-[10].

$$
\Delta T_{\text {Surface }} \approx \int_{0}^{\infty} \int_{0}^{a} 2 \pi r J^{2}(r, t) d r d t / \sigma \pi a^{2} c
$$

In Eq. (11), $c$ is the specific heat. Equation (11) was derived assuming that there is no heat transport during the time of the lightning pulse. The results are shown in table 1 for the approximate temperature rise at the surface when excited by the Heidler and Double Exponential lightning pulses. The results are given for rebar sized 
Table 1. Approximate temperature rise at the surface of the conductor when excited by a lightning pulse.

\begin{tabular}{|l|c|c|c|c|c|}
\hline & $\mathrm{Cu}$ & Sat. & Eff. $\mu \mathrm{r}$ & Unsat. & B.W. \\
\hline Heidler & $0.6^{\circ} \mathrm{C}$ & $6^{\circ} \mathrm{C}$ & $6^{\circ} \mathrm{C}$ & $90^{\circ} \mathrm{C}$ & $100^{\circ} \mathrm{C}$ \\
\hline Dbl. Exp. & $0.4^{\circ} \mathrm{C}$ & $3^{\circ} \mathrm{C}$ & $4^{\circ} \mathrm{C}$ & $70^{\circ} \mathrm{C}$ & $60^{\circ} \mathrm{C}$ \\
\hline
\end{tabular}

conductors made of copper, saturated and unsaturated steel, an effective permeability with the conductivity of steel 1008, and the copper bond wire. It was found for the nonlinear steel 1008 rebar that the impedances were approximated well after the current was appreciable by using $\mu_{r} \approx 2$. This is referred to as Eff. $\mu_{r}$ in table 1 .

We can see from the results in table 1 that the temperature change at the surface is negligible for copper, saturated steel, and effective permeability. However for the unsaturated steel and the bond wire the temperature change is not negligible and would result in a small change in parameters that we treated as constants with temperature, such as the conductivity. For the analysis performed in this paper we have assumed only one return stroke for the lightning strike, however as many as 25 return pulses can occur per strike [3], which would cause the temperature at the surface to increase more. Similarly, if the lightning strike was an extreme positive strike rather than the negative strike assumed in this report, the peak lightning current could be $500 \mathrm{kA}$ or greater [3]. Closely related to the temperature rise of the cylindrical conductors is the temperature rise and forces that result at the joints of the bond wires [11]-[13]. An area of future research is to ensure that the large lighting currents do not cause the bond wires to rip out of the joints used to connect them to the facility.

\section{ACKNOWLEDGEMENTS}

The authors would like to thank Constantine Hrousis and Ronald Streit of LLNL for supporting this research. We would also like to thank Jay Javedani for insightful discussions regarding this work and Monica Garcia for administrative support.

\section{REFERENCES}

[1] M.P. Perkins, M.M. Ong, R.D. Speer, and C.G. Brown Jr., "Analysis of a Small Loop Antenna with Inductive Coupling to Nearby Loops," accepted by IEEE Trans. on Electromagnetic Compatibility.
[2] M.P. Perkins, M.M. Ong, E.W. Crull, and C.G. Brown Jr., "Modeling Techniques used to Analyze Safety of Payloads for Generic Missile Type Weapons Systems During an Indirect Lightning Strike," Proc. Of $31^{\text {st }}$ Ann. Antenna Measurement Techniques Assoc. Symp., 2009.

[3] M.A. Uman, V.A. Rakov, J.O. Elisme, D.M. Jordan, C.J. Biagi, J.D. Hill, "Update Direct-Strike Lightning Environment for Stockpile-to-Target Sequence,” LLNLSR-407603, 2008.

[4] M. Rosu, S. Stanton, J.R. Brauer, and Z.J. Cendes, "Complete Nonlinear Magnetic-Thermal-Stress Design of Radial Field Multipole NdFeB Permanent Magnet Cylinder,” IEEE Int. Conf. Electric Machines and Drives, 2005, p. 131.

[5] P. Zhou, D. Lin, W.N. Fu, B. Ionesu, and Z.J. Cendes, "A General Co-Simulation Approach for Coupled Field-Circuit Problems,” IEEE Trans. on Magnetics, vol. 42, no. 4, p. 1051, 2006.

[6] B.E. Fridman, "Transients in Pulsed Electrical Circuits with Massive Conductors,” $15^{\text {th }}$ IEEE Pulsed Power Conf., 2005, p. 709.

[7] O.M. Gatous, and J.P. Filho, "A New Formulation for Skin-Effect Resistance and Internal Inductance Frequency-Dependent of a Solid Cylindrical Conductor,” IEEE/PES Trans. \& Dist. Conf. \& Exp., 2004, p. 919.

[8] J.L. Maksiejewski, "Calculation of Losses in Conductors Due to Chopped Impulse Currents, Taking the Skin Effect into Account," IEE Proc. Science, Meas. \& Tech., vol. 144, no. 3, p. 111, 1997.

[9] J.L. Maksiejewski, "Losses in Conductors Due to Current Surges Taking the Skin Effect into Account," IEE Proc. Science, Meas. \& Tech., vol. 137, no. 2, p. 80, 1990. [10] M.P. Paisios, C.G. Karagiannopoulos, and P.D. Bourkas, "Estimation of the Temperature Rise in Cylindrical Conductors Subjected to Heavy 10/350 $\mu$ s Lightning Current Impulses,” Electric Power Systems Research, vol. 78, no. 1, p. 80, 2007.

[11] A.D. Polykrati, M.P. Paisios, C.G. Karagiannopoulos, and P.D. Bourkas, "Model for Temperature Estimation of Electric Couplings Suffering Heavy Lightning Currents,” IEE Proc. Generation, Transmission, and Distrib., vol. 151, no. 1, p. 90, 2004.

[12] J.M. Tobias, "Testing of Ground Conductors with Artificially Generated Lightning Current,” IEEE Trans. on Industry Applications, vol. 32, no. 3, p. 594, 1996.

[13] J.L. Maksiejewski, "Thermal Response of Coated Steel Conductors Under Surge Currents Taking the Skin Effect into Account,” IEE Proc. Science, Meas. \& Tech., vol. 143, no. 6, p. 389, 1996. 